Zeitschrift für Physik 211, 418 (1968)

\title{
Erratum
}

\section{H. Pelikan: Theory of Coupled Mode Oscillation in a Laser with Axial Magnetic Field}

Z. Physik 201, 523 (1967)

Received February 17, 1968

The splitting of Eq. (2.8) into the equation for the frequency (3.4) contains a sign error. Eq. (3.4) should read

$$
\Omega_{ \pm}+\dot{\varphi}_{ \pm}=\omega_{ \pm} \pm \Delta \kappa \frac{B_{\mp}}{B_{ \pm}} \sin \psi \delta_{\omega_{+}, \omega_{-}}+\sigma_{ \pm}-\rho_{ \pm} B_{ \pm}^{2}-\tau_{ \pm} B_{\mp}^{2} .
$$

This sign error also occurs in the Eqs. (4.2) and (5.1). They should read

$$
\begin{aligned}
\dot{\varphi}_{ \pm}= & \pm \Delta \kappa \frac{B_{\mp}}{B_{ \pm}} \sin \psi \delta_{\omega_{+}, \omega_{-}} \\
= & \pm \Delta \kappa \frac{B_{\mp}}{B_{ \pm}} \sin \left[\left(\Omega_{-}-\Omega_{+}\right) t+\varphi_{-}-\varphi_{+}\right] \delta_{\omega_{+}, \omega_{-}} \\
\dot{\psi}= & \Omega_{-}-\Omega_{+}+\dot{\varphi}_{-}-\dot{\varphi}_{+} \\
= & \omega_{-}-\omega_{+}+\sigma_{-}-\sigma_{+}-\left(\rho_{-} B_{-}^{2}-\rho_{+} B_{+}^{2}\right)-\left(\tau_{-} B_{+}^{2}-\tau_{+} B_{-}^{2}\right) \\
& -\Delta \kappa\left(\frac{B_{+}}{B_{-}}+\frac{B_{-}}{B_{+}}\right) \sin \psi \delta_{\omega_{+}, \omega_{-}} \\
= & \Omega_{-}-\Omega_{+}-a \sin \psi \delta_{\omega_{+}, \omega_{-}}
\end{aligned}
$$

where

$$
a=+\Delta \kappa\left(\frac{B_{+}}{B_{-}}+\frac{B_{-}}{B_{+}}\right) \text {. }
$$

There is now frequency locking in the case $B_{+}=B_{-}$too, in agreement with M. Sargent III, W. E. LamB, R. L. Fork, Phys. Rev. 164, 436, 450 (1967).

Dipl.-Phys. H. Pelikan

II. Inst. f. Theoret. Physik der TH 7000 Stuttgart 1, Herdweg 77 